\title{
Simple solvation potential for coarse-grained models of proteins
}

\author{
A. Bhattacharyay*, A. Trovato ${ }^{\dagger}$, F. Seno \\ Dipartimento di Fisica "G.Galilei", Università degli Studi di Padova, via F. Marzolo 8, 35131 Padova, Italy
}

(Dated: November 13, 2018)

\begin{abstract}
We formulate a simple solvation potential based on a coarsed-grain representation of amino acids with two spheres modeling the $C_{\alpha}$ atom and an effective side-chain centroid. The potential relies on a new method for estimating the buried area of residues, based on counting the effective number of burying neighbours in a suitable way. This latter quantity shows a good correlation with the buried area of residues computed from all atom crystallographic structures. We check the discriminatory power of the solvation potential alone to identify the native fold of a protein from a set of decoys and show the potential to be considerably selective.
\end{abstract}

Keywords: Protein folding, structure prediction, solvation, buried area

\footnotetext{
* arijit@pd.infn.it

$\dagger$ antonio.trovato@pd.infn.it

$\ddagger$ flavio.seno@pd.infn.it
} 


\section{INTRODUCTION}

The prediction of three dimensional structure of the native state of proteins from the knowledge of their sequence of amino-acids can only be achieved if the interaction potentials among various parts of the peptide chain in the presence of solvent molecules are known to some extent. In fact, approaches to protein structure prediction are based on the thermodynamic hypothesis that the native state of a protein is the state of lowest free energy under physiological conditions [1]. Thus, the computation of the energy of a sequence in a given conformation would be the fundamental step toward the solution of the protein folding problem [2]. A rigorous approach [3] from "first principles" taking into account the quantum mechanics of a huge number of atoms constituting the protein is not practical and beyond actual computational capabilities. An usual way [4] to avoid dealing with too many microscopic degrees of freedom is to introduce a reduced representation of proteins in which each amino acid is represented by one or a few interaction sites. The main difficulty with such simplified representations is the need of an effective energy that captures, at least, the essential qualitative physical and chemical features of the folding process. This involves choosing the right contributions to the energy function as well as determining the related potentials [see for example 2,26-30].

Since the classical work of Kauzmann [5] it is evident [6] that the hydrophobic effect is one of the leading forces in the folding process. Hydrophobicity arises because the side-chain of some amino acids is not able to form hydrogen bonds with the surrounding water, and as a consequence its solubility is low. Gordon et al. [7] have reported a free energy difference associated with exposing such groups to water which is comparable with the strength of hydrogen bonds. Moreover, several experiments $[8,9,10,11]$ have shown that non specific interaction and placement of hydrophobic residues is a critical determinant of protein structure.

Starting from the seminal work of Eisenberg and McLachlan [12] several efforts have been produced to model the hydrophobic effects [13-17] but a precise and computationally efficient method is still lacking. The purpose of this work is to cover this gap by developing a solvation energy for a coarse-grained model of a protein which is more accurate than the other existing ones but still is easy for numerical implementation in folding simulations. The energy function we propose is the sum over all the amino-acids of a contribution given by a factor proportional to the effective number of particles which are screening the amino-acid from its aqueous surroundings multiplied by an amino acid specific parameter measuring its hydrophobicity degree. These parameters are calculated through a statistical analysis on a training set of proteins. 
An important test to validate any energy function is to check its ability to recognize native structures among a large number of well constructed decoys [18-21]. A number of standard decoy sets, consisting of the native structure plus a large ensemble of simulated protein-like structures have been established for benchmarking purposes [22]. The performance of our method on different test sets is better than other solvation energies $[16,17]$ and is comparable to scoring functions which implicitly include other energetic contributions such as hydrogen bond, torsion angle and van der Waals potentials $[23,24]$.

\section{METHODS}

In our model each residue is simplified as follows. The $C^{\alpha}$ atoms are represented by a sphere of radius $1.9 \AA$. The specific characteristic of each amino acid is captured by its side-chain which we again model as a sphere. The center of this sphere is located at the geometric center, $\mathbf{X}_{\mathbf{c}}$, of all side chain atoms including the $C_{\beta}$ atom, namely at:

$$
\mathbf{X}_{c}=\frac{\sum_{l=1}^{m} \mathbf{X}_{l}}{m}
$$

where $\mathbf{X}_{\mathbf{l}}$ are the positions of all the atoms in the given side chain and $m$ is the total number of atoms in the side chain. The radius $r_{c}$ of the side-chain sphere is defined as the root mean square deviation of the position of the side-chain atoms of that residue from $\mathbf{X}_{\mathbf{c}}$. Relative changes in positions of side chain atoms of a particular residue in various proteins are reflected in the corresponding change in position of the side-chain center and in some fluctuation of the side change radius. Since modeling side chains as spheres is a gross simplification of side chain geometry, the slight overlap of different sphere pairs can occur. Note that throughout this paper we do not enforce any steric constraint.

Our main purpose is to find a way within this representation to compute the effective number $N_{i}^{a}$ of spheres (both $C^{\alpha}$ and side chains) which are burying the side chain of the amino-acid of type $a$, located at position $i$ along the chain, from the solvent. This number will then be used as an estimation of the buried area of residue $i$ in order to define a solvation energy in this coarse-grained perspective. Let us call $D_{j k}$ the distance between two generic spheres $j$ and $k$ (note the difference between indexes $j, k$ going through both $C^{\alpha}$ and side chains and residue index $i$ going only through side chain spheres). All other spheres in the chain, either $C^{\alpha}$ or side chains, contribute to $N_{i}^{a}$ through the relation: 


$$
N_{i}^{a}=\sum_{\substack{j \notin i \\ j \notin i \pm 1}} B_{i j} S_{i j}
$$

where the sum is running only through spheres belonging to residues not adjacent to $i$. $B_{i j}$ represents the bare contribution by sphere $j$ to the protection of side chain $i$ from water and it is equal to 1 if the distance $D_{i j}$ is smaller than $R_{i j}=r_{i}+r_{j}+\frac{r_{\text {water }}}{2}\left(r_{\text {water }}=1.4 \stackrel{\circ}{A}\right.$ is the radius of a water molecule) or $\frac{R_{i j}}{D_{i j}}$ otherwise. The threshold $R_{i j}$ has been chosen to discriminate between two regimes. If $D_{i j}<R_{i j}$, a portion of the surface area of side chain $i$ is screened from contact with water molecules, since the latter can only marginally accomodate between side chain $i$ and sphere $j$ (see Fig. 2). If $D_{i j}>R_{i j}$, this constraint disappears but in order to take into account fluctuations, due to the varying geometry of side chains and to the simple assumption of spherical geometry, we assume the screening effect to be still present and decaying as $\frac{1}{d}$.

$S_{i j}$ instead takes into account the fact that the screening effect of sphere $j$ on side chain $i$ can be already accounted for by the presence of other spheres of the chain. We define $S_{i j}$ as

$$
S_{i j}=\prod_{\substack{k \neq j \\ k \notin i, i \pm 1}} S_{i j}^{k}
$$

where we multiply the individual contributions $S_{i j}^{k}$ of different spheres to the overlap of screening effects, given by

$$
S_{i j}^{k}=\left\{\begin{array}{ll}
\left(1-M_{i j k}\right) \frac{r_{j}}{r_{k}} & \text { if } M_{i j k}>0.7 \\
1 & \text { if } M_{i j k} \leq 0.7 \text { or } D_{i j} \leq D_{i k}
\end{array}\right\}
$$

The coefficients $M_{i j k}$ are defined as

$$
M_{i j k}=\frac{D_{i j}}{D_{i k}+D_{k j}}
$$


The $M_{i j k}=1$ limit corresponds to a complete overlap of the screening effects (Fig.2c) so that in this case $S_{i j}^{k}=0$; sphere $j$ is not contributing effectively in screening side chain $i$ from water molecules more than sphere $k$ is already doing. It is easy to demonstrate that when three spheres of equal size are in contact with each other, $M_{i j k}$ is equal to 0.5 (e.g. see Fig.2a). In such a situation no sphere is actually obstructing the other two to come in complete contact and there is no overlap of the screening effects. Starting from such a limiting geometry, when sphere $k^{\text {th }}$ is inserting between the other two (Fig.2b) the value of $M_{i j k}$ is increasing. The choice of 0.7 as the lower limit to measure the screening overlap has been made based upon an optimization procedure. The factor $\frac{r_{j}}{r_{k}}$ takes into account the fact that the bigger $r_{k}$ the more overlapping the screening effects of $j$ and $k$, and therefore the smaller $S_{i j}^{k}$. It is interesting to note that statistics of side chain sizes, based on our coarse-grained representation, reveals that the ratio $r_{j} / r_{k}$ is generally less than 3 . So, $1 \geq M_{i j k}>0.7$ never let contributions to $S_{i j}^{k}$ be bigger than unity.

To test our method we use the Top500H database [31] of non-redundant protein structures. It is a hand-curated set of 500 high-resolution structures all solved by X-ray crystallography to $1.8 \AA$ or better resolution. From this database, only those 220 proteins which do not have any discontinuity in the chain have actually been selected. The analysis of this proteins shows that the effective number of contacts is ranging from 0 to about 12 (see Fig. 3). We took 10 to be the upper limit in all further calculation since it was already shown to be roughly the number of neighbours with which an amino-acid becomes buried (see, e.g. [17,32]). Our way of taking into account the overlap of different screening effects could indeed produce some overcounting and whenever the counting of $N_{i}^{a}$ is above 10 , it is put equal to it. To check the correspondence between $N_{i}^{a}$ and buried area, we have calculated the buried area of residues of these proteins by using on-line available program 'GETAREA 1.1' [34] (URL: http://www.scsb.utmb.edu/cgi-bin/get_a_form.tcl). The number $B_{i}^{a}$ that we use from this program is the percentage of buried side-chain surface area with respect to the "random coil" value per residue. The "random coil" value for residue is the average solvent-accessible surface area of $a$ in the tripeptide $G l y-a-$ Gly in an ensemble of 30 random conformations. $B_{i}^{a}$ varies from $0 \%$ (completely exposed) to $100 \%$ (fully buried) and it is plotted in Fig.3 against $N_{i}^{a}$ for all amino-acids of a set of proteins (see Results for discussions). The average buried area $\bar{B}^{a}$ of each residue kind is displayed in Table A.

In order to develop the solvation energy we consider the amino-acids to be hydrophobic if their average buried area is more than $60 \%$, otherwise we take them to be polar. The solvation energy parameters $E_{a}$ for different residue types, listed in Table A, are obtained through the relation: 


$$
E_{a}=\left(60-\bar{B}^{a}\right) /\left(\bar{B}_{\max }-60\right)
$$

where $\bar{B}_{\max }=\max \left\{\bar{B}^{a}\right\}=86.90 \%$. In our coarse-grained representation the overall solvation energy is then obtained summing contribution from all residues, negative for hydrophobic ones $\left(E_{a}<0\right)$, and positive otherwise, which are proportional to the residue buried area $N_{i}^{a}$ evaluated from Eq. (10):

$$
E_{\text {solv }}=\sum_{i} E_{a} \times N_{i}^{a}
$$

Note that no solvation energy is associated to $G L Y$ in our model, but it takes part into the calculation of solvation energy of other residues by virtue of its $C^{\alpha}$ sphere.

Five different performance measures have been applied to assess the ability of our solvation potential in discriminating the native structure from native like ones. The Rank1 measure is the one which is more direct in the sense it tells us about the position of the native state among the decoys in an arrangement of increasing order of their energy. So, Rank1 being equal to 1 means the native fold is the lowest energy conformation among the decoys. We also employ the $Z$-score which is defined as

$$
Z-\text { score }=\frac{E_{\text {native }}-\bar{E}}{\sigma}
$$

where, $E_{\text {native }}$ is the energy of the native fold and $\bar{E}$ is the average energy of decoys with a standard deviation $\sigma$. We also employ additional performance measures to understand the discriminatory capacity of our potential for native like structures rather than oly for the native fold. To assess such properties we use $\log P B 1, \log P B 10$ and F.E. (fraction enrichment) [33] measures to see how the present potential fares. $\log P B 1$ is the $\log$ probability of selecting the best scoring conformation and is given by 


$$
\log P B 1=\log _{10}\left(\frac{R_{i}}{p}\right)
$$

where $R_{i}$ is the $R M S D$ rank of the best scoring conformation in $p$ decoys. $\log P B 10$ is the probability of selecting the lowest $R M S D$ conformation among the 10 best scoring conformations $\left(R_{i}=\min \left\{R_{1}, \ldots, R_{10}\right\}\right)$. The $F$.E. or fraction enrichment is the percentage of the top $10 \%$ lowest $R M S D$ conformations in the top $10 \%$ best scoring conformations.

\section{RESULTS}

Our first test aims to verify the existence of a correlation between the method, presented in the previous section, of counting effective neighbours $\left(N_{i}^{a}\right)$ of an aminoacid and its buried area. To perform this test, we have used the Top500H database [31] of non-redundant protein structures selecting only those proteins which do not have any discontinuity in the chain. Fig.3 shows a plot of the calculated buried area, obtained using GETAREA 1.1, of different residues in their native conformations versus their $N_{i}^{a}$. The correlation turns out to be quite good (coefficient of correlation $=$ $0.89)$.

The crucial test for our method consists, however, in assessing the ability of the solvation energy based on Eq. (2) to discriminate between native states and not native decoy conformations for the same sequence. A good decoy set must include a large number of conformations, some near native and other that are native-like in all respects except the overall folded conformation and this set should be generated independently from the evaluated scoring mechanisms to avoid bias toward any particular selection methodology. Our solvation potential has been tested on four such standard decoy sets: 4state_reduced, lattice_ssfit, lmds sets from the Decoys 'R' Us web sites (http//:dd.stanford.edu and http//:ddcompbio.washington.edu) and the Rosetta decoys from the Baker laboratory site (http//:depts.washington.edu/bakerpg).

In Table B we arrange the average scores for the different sets whereas the detailed account of the individual scores for every target in various decoy sets are given in Tables C-F. This detailed scores are more revealing than the average ones due to the fact that some targets which are scoring particularly bad due to some specific reasons can affect the average score.

For the 4 state_reduced, we observe that $\operatorname{Rank} 1=1$ four times out of $\operatorname{seven}(4 / 7$.) It is 2 for the protein 2 cro 
whereas 5 and 4 for the proteins $4 p t i$ and $4 r x n$, respectively. Protein $4 p t i$ has three disulphide bonds which might be in conflict with the optimum arrangement on the basis of solvation energy alone. This performance can be compared with other two solvation potentials: the one called Chebyshev-expanded hydrophobic potential $(C H P)$ introduced by Fein, Xia and Levitt [17] and the one introduced by Jones [16] (SOLV) used by Tosatto [25] to formulate a combined potential function called FRST. The discriminatory predictions of $C H P, S O L V$ and FRST as well (although is not a solvation potential) are reported in table $\mathrm{G}$ and $\mathrm{H}$ for the available sets and with the available information. For the 4state_reduced set, both $C H P$ and $S O L V$ get Rank $=1$ only in one case and both methods with a worse average value for the parameter $Z$-score.

For the lattice_ssfit set of decoys we obtain Rank1 $=1$ in 6 cases out of 8 . Again the performance is much better of $S O L V$ (there are not available data for $C H P$ ) which has $R a n k 1=1$ in 4 cases out of 8 , and worse values for the $Z$-score, $\log P B 1, \log P B 10$ and F.E. parameters. In this set, the targets for which our method gives $\operatorname{Rank} 1 \neq 1$ are the proteins $1 d k t-A$ and $1 t r l-A$ : these two proteins are a part of a whole chain. The missing parts might have residues which contribute to the count of $N_{i}^{a}$. Their absence can drastically reduce the efficiency of the methods.

For the set of decoy $l m d s, R a n k 1=1$ in 4 proteins out of 8 . The proteins $1 b 0 n$ - $B$ and $1 f c 2$ are the ones which show almost no selection on the basis of this solvation potential because they are short chains of hetero-dimers. Other two, 2 ovo and $4 p t i$, have three disulfide bonds. Interestingly, $4 p t i$ has a Rank1 measure of the same order also in the decoy sets 4state_reduced and Rosetta. Again the performance is much better than for the other two solvation energy potentials.

For the set Rosetta, Rank $1=1$ in 17 cases out of 23 . In this collection of decoys, there are proteins which have residues clipped from the chain end and thus decoys are considerably shorter than the actual protein. We have selected those targets from Rosetta which have at most up to 8 residues clipped from the end of a chain (in Table F the numbers within bracket just after the target name are the numbers of residues clipped). We are not considering those having more residues clipped since it can affect proper calculation of solvation energy. It is important to note that the targets for which the Rank1 measure is not very good are mainly those which have a large number of residues clipped, even though some of the latter have Rank $1=1$. We can not compare our method on this decoys set with CHP and SOLV since these data are not available. Nevertheless, we make a comparison with an all atom-atom contact scoring energy proposed by McConey et al. [23] which has a record for Rank $=1$ of 19 out of 23 with a $Z-$ score $=-3.6$, performances which are just slightly better of ours. Keeping in mind that the approach in [23] is 
taking care in detail of various interactions with more than 28000 parameter it turns out that our solvation energy has a very high degree of confidence in discriminating native state with respect to other methods.

For all the set in which is possible to make a comparison the full algorithm $F R S T$ is performing better than our method, although sometimes the values of the less selective parameters $\log P B 1, \log P B 10$, and $F . E$ are comparable. This is a consequence of the fact that FRST is an algorithm which combine four different knowledge based potentials. In addition to the solvation potential $(S O L V)$, there are the pairwise, the hydrogen bond and the torsion angle potentials. The results we presente here suggest that, regarding the development of refined methods for structure recognition, it should be convenient to include our method rather than SOLV in the FRST algorithm.

\section{DISCUSSION}

In the present work we have proposed a simple and efficient method to estimate the buried area of a side chain group by counting the effective number of atoms which are screening it from water. This evaluation builds on the assumption of spherical symmetry of the interacting side chain groups. It corrects for this oversimplification by allowing the screening effect to be considered beyond the range dictated by sphere sizes and by considering the threebody contribution to its cooperativity. The resulting quantity is well related to the buried area and it can be used to compute a solvation energy which is based just on 20 energy solvation parameters, one for each kind of amino-acid. This solvation energy works pretty well, better than any other solvation energy, in recognizing native structures among set of well constructed, alternative decoys. The use of a small number of parameters makes our solvation potential more physically transparent than other elaborate knowledge based energy functions with a much larger parameter space.

The targets which are parts of larger chain, or have a good number of residues clipped from the chain end or having many disulfide bonds etc are generally those for which the present solvation potential fails to identify the native fold.

Our potential does not implicitly include any other interaction than the solvation effect, so it should be easily improved by combining it with other interaction terms. Moreover, since the model we use to represent amino-acids is quite simplified, the approach can be easily implemented in ab-inito simulation of protein folding. 


\section{ACKNOWLEDGMENTS}

We thank A. Maritan and S. Tosatto for sitmulating discussion. This work was supported by PRIN 2005 prot. 2005027330 and the Program "Progetti di Ateneo" of Padua University.

[1] Anfinsen C. Principles that govern the folding of protein chains. Science 1973;181:223-230.

[2] Lazaridis T, Karplus M. Effective energy functions for protein structure prediction. Current Opinion in Structural Biology 2000;10:139-145 .

[3] Van Gusteren W. Computing Simulation of Biomolecules Systems. ESCOM Science Publishers, B.V. Leiden Netherlands 1989.

[4] Levitt M. A simplified representation of protein conformations for rapid simulation of protein folding. J Mol Biol $1976 ; 104: 59$.

[5] Kauzmann W. Advan Prot Chem 1959;14:1.

[6] Dill Ken A. Dominant forces in protein folding. Biochemistry 1990;29:7133-7155.

[7] Gordon DB, Marshall SA, Mayo SL. Energy functions for protein design. Current Opinion in Structural Biology 1999;9:509-513

[8] Kamtekar S, Shiffer JM, Xiong HY, Babik JM, Hecht MH. Protein design by binary patterning of polar and non polar amino-acids. Science 1993;262:1680-1685.

[9] Lum WA, Sauer RT. Alternative packing arrangements in the hydrophobic core of represser. Nature 1989;339:31-36.

[10] Sali DS, Bycroft M, Fersht AR. Surface electrostatic interactions contribute little to stability of barnase. J Mol Biol 1991;210:779-788.

[11] Shortle D, Stites WE, Meeker AK. Contributions of the large hydrophobic amino acids to the stability of staphylococcal nuclease. Biochemistry 1990;29:8033-8041.

[12] Eisenberg D, McLachlan AD. Solvation energy in protein folding and binding. Nature 1989;319:199-203.

[13] Cassari G, Sippl MJ. Structure-derived hydrophobic potential : Hydrophobic potential derived from X-ray structures of globular proteins is able to identify native folds. J Mol Biol 1992;224:725-732.

[14] Delarue M, Koehl P. Atomic environment energies in proteins defined from statistics of accessible and contact surface areas. J Mol Biol 1995;249:675-690.

[15] Huang ES, Subbiah S, Levitt M. Recognizing Native Folds by the Arrangement of Hydrophobic and Polar residues. J Mol Biol 1995;252:709-720.

[16] Jones DT. GenThreader: an efficient and reliable protein fold recognition method for genomic sequences. J Mol Biol 
1999;287:797-815.

[17] Fain B, Xia Y, Levitt M. Determination of optimal Chebyshev expanded hydrophobic discrimination function for globular proteins. IBM J Res Dev 2001;45:525-532.

[18] Simons KT, Ruczinski I, Kooperberg C, Fox BA, Bystroff C, Baker D. Improved recognition of native-like protein structures using a combination of sequence-dependent and sequence-independent features of proteins. Proteins 1999;34:82-85.

[19] Holm L,Sander C. Evaluation of protein models by atomic solvation preference. J Mol Biol 1992;225:93-105.

[20] Park B, Levitt M. Energy Functions that Discriminate X-ray and Near-native Folds from Well-constructed Decoys. J Mol Biol 1996;258:367-392.

[21] Xia Y, Huang ES, Levitt M, Samudrala R. Ab initio construction of protein tertiary structures using a hierarchical approach. J Mol Biol 2000;300:171-185.

[22] Samudrala R, Levitt M. Decoys 'R' Us: a database of incorrect conformation to improve protein structure prediction. Protein Science 2000;9:1399-1401.

[23] McConkey BJ, Sobolev V, Edelman M. Discrimination of native protein structures using atom-atom scoring. Proceeding of the National Academy of Science USA 2003;100:3215-3220.

[24] Summa CM, Levitt M, DeGrado W. An atomic environment potential for use in structure prediction. J Mol Biol $2005 ; 352: 986-1001$.

[25] Tosatto SCE. The Victor/FRST function for model quality estimation J Comp Biol 2005;12:1316-1327

[26] Maiorov VN, Crippen GM. Contact potential that recognizes the correct folding of globular proteins. J Mol Biol $1992 ; 227: 876-888$.

[27] Miyazawa S, Jernigan RL. Estimation of effective interside contact energies from protein crystal structures: quasichemical approximation. Macromolecules 1985;18:534-552.

[28] Mirny LA, Shakhnovich EI. How to derive a protein folding potential? a new approach to a old problem. J Mol Biol $1996 ; 264: 1164-1179$.

[29] Sippl MJ. Knowledge based potentials for proteins. Curr Opin 1995;229-235.

[30] Seno F, Maritan A, Banavar JR. Interaction potentials for protein folding. Proteins 1998;30:244-248.

[31] Lovell Sc, Davis IW, Arendall WBr, De Bakker PI, Word JM, Prisant MG, Richardson JS, Richardson DC. Structure validation by Calpha geometry: phi, psi and Cbeta deviation. Proteins 2003;50:437-450.

[32] Viswanadhan VN. Hydrophobicity and residue-residue contacts in globular proteins. Int J Biol Macromol 1987;9:39-48.

[33] Wang K, Fain B, Levitt M, Samudrala R. Improved protein structure selection using decoy dependent discriminatory functions. BMC Struct Biol 2004;4:8.

[34] Fraczkiewicz R, Braun W. Exact and efficient analytical calculation of the accessible surface areas and their gradients for macromolecules. J. Comp. Chem. 1998; 19:319. 
Table A: Data showing average measure of buried area for Amino acids in native state's database

\begin{tabular}{|c|c|c|}
\hline Amino acid & $\bar{B}^{a}$ & $E_{a}$ \\
\hline ALA & 71.55 & -0.39 \\
\hline $\mathrm{ARG}$ & 59.57 & 0.01 \\
\hline $\mathrm{ASN}$ & 58.77 & 0.04 \\
\hline $\mathrm{ASP}$ & 55.87 & 0.14 \\
\hline CYS & 89.80 & -1.00 \\
\hline GLN & 57.46 & 0.09 \\
\hline GLU & 50.44 & 0.32 \\
\hline HIS & 70.12 & -0.34 \\
\hline ILE & 86.90 & -0.90 \\
\hline LEU & 85.48 & -0.86 \\
\hline LYS & 44.30 & 0.53 \\
\hline MET & 81.92 & -0.74 \\
\hline PHE & 86.82 & -0.90 \\
\hline PRO & 57.34 & 0.09 \\
\hline SER & 61.94 & -0.07 \\
\hline THR & 65.91 & -0.20 \\
\hline $\mathrm{TRP}$ & 84.95 & -0.84 \\
\hline TYR & 80.36 & -0.68 \\
\hline VAL & 85.36 & -0.85 \\
\hline
\end{tabular}


Table B: Average performance measures

\begin{tabular}{l|ccccc} 
Decoy Set & Rank 1 & $Z$-score & $\log P B 1$ & $\log P B 10$ & F.E. \\
\hline 4state_reduced & $4 / 7$ & -2.89 & -1.19 & -1.88 & 0.28 \\
lmds & $4 / 8$ & -2.75 & -0.48 & -1.36 & 0.14 \\
lattice_ssfit & $6 / 8$ & -4.06 & -0.41 & -1.55 & 0.12 \\
rosetta & $17 / 23$ & -3.44 & -0.54 & -1.65 & 0.16 \\
\hline Total/average & $31 / 46$ & -3.28 & -0.85 & -1.61 & 0.17 \\
\hline
\end{tabular}

Table C: Performance Measure for 4state_reduced

\begin{tabular}{l|ccccc} 
Target & Rank 1 & $Z$-score & $\log P B 1$ & $\log P B 10$ & F.E. \\
\hline $1 \mathrm{ctf}$ & 1 & -3.53 & -2.10 & -2.10 & 0.55 \\
$1 \mathrm{r} 69$ & 1 & -3.68 & -1.93 & -2.53 & 0.27 \\
$1 \mathrm{sn} 3$ & 1 & -2.52 & -0.27 & -1.09 & 0.10 \\
$2 \mathrm{cro}$ & 2 & -3.01 & -1.26 & -2.53 & 0.32 \\
3 icb & 1 & -2.26 & -2.12 & -2.12 & 0.46 \\
$4 \mathrm{pti}$ & 5 & -2.51 & -0.50 & -1.48 & 0.06 \\
$4 \mathrm{rxn}$ & 4 & -2.71 & -0.15 & -1.88 & 0.20 \\
\hline Total/average & $4 / 7$ & -2.89 & -1.19 & -1.88 & 0.28 \\
\hline
\end{tabular}


Table D: Performance Measure for lmds (* excluded from the calculation of averages. We have not considered the score of the targets $1 b 0 \mathrm{nB}$ and $1 f c 2$ while calculating the averages. )

\begin{tabular}{l|ccccc} 
Target & Rank 1 & $Z$-score & $\log$ PB1 & $\log$ PB10 & F.E. \\
\hline 1b0n-B* & 439 & 1.18 & -0.01 & -0.32 & 0.04 \\
$1 \mathrm{ctf}$ & 1 & -3.42 & -0.36 & -0.77 & 0.08 \\
$1 \mathrm{fc} 2^{*}$ & 409 & 0.91 & -0.31 & -2.22 & 0.07 \\
1igd & 1 & -2.87 & -0.25 & -1.39 & 0.14 \\
1shf-A & 1 & -2.90 & -0.14 & -1.09 & 0.09 \\
2cro & 1 & -3.42 & -0.60 & -1.37 & 0.18 \\
2ovo & 16 & -1.67 & -1.00 & -1.46 & 0.14 \\
4pti & 6 & -2.24 & -0.53 & -2.06 & 0.20 \\
\hline Total/average & $4 / 8$ & -2.75 & -0.48 & -1.36 & 0.14 \\
\hline
\end{tabular}

Table E: Performance Measure for lattice_ssfit

\begin{tabular}{|c|c|c|c|c|c|}
\hline Target & Rank 1 & $Z$-score & $\log \mathrm{PB} 1$ & $\log \mathrm{PB} 10$ & F.E. \\
\hline $1 \mathrm{ctf}$ & 1 & -5.04 & -0.06 & -0.68 & 0.14 \\
\hline 1 beo & 1 & -3.67 & -0.36 & -1.13 & 0.12 \\
\hline $1 \mathrm{dkt}-\mathrm{A}$ & 8 & -2.73 & -0.20 & -2.35 & 0.11 \\
\hline 1 fca & 1 & -7.38 & -2.45 & -2.45 & 0.09 \\
\hline 1nkl & 1 & -4.54 & -0.01 & -2.52 & 0.08 \\
\hline 1pgb & 1 & -4.01 & -0.10 & -0.51 & 0.10 \\
\hline 1 trl-A & 101 & -1.61 & -0.12 & -0.98 & 0.18 \\
\hline $4 \mathrm{icb}$ & 1 & -3.50 & -0.01 & -1.75 & 0.12 \\
\hline Total/average & $6 / 8$ & -4.06 & -0.41 & -1.55 & 0.12 \\
\hline
\end{tabular}


Table F: Performance Measure for rosetta (* excluded from the calculation of averages)

\begin{tabular}{|c|c|c|c|c|c|}
\hline Target & Rank 1 & $Z$-score & $\log P B 1$ & $\log \mathrm{PB} 10$ & F.E. \\
\hline 1aa2(3) & 1 & -4.18 & -0.65 & -1.70 & 0.15 \\
\hline $\operatorname{1acf}(2)$ & 1 & -5.34 & -0.31 & -2.15 & 0.23 \\
\hline $1 \mathrm{bdo}(5)$ & 1 & -3.81 & -0.03 & -1.55 & 0.14 \\
\hline $1 \operatorname{cc} 5(7)^{*}$ & 407 & -0.30 & -0.12 & -1.06 & 0.12 \\
\hline $1 \operatorname{csp}(3)$ & 1 & -2.73 & -0.18 & -2.52 & 0.20 \\
\hline $1 \operatorname{ctf}(1)$ & 1 & -3.49 & -0.06 & -1.34 & 0.14 \\
\hline 1eca(4) & 1 & -3.67 & -0.21 & -2.00 & 0.13 \\
\hline $1 \operatorname{erv}(0)$ & 1 & -4.20 & -0.56 & -3.00 & 0.21 \\
\hline $1 \mathrm{kte}(5)$ & 1 & -2.67 & -1.55 & -1.55 & 0.12 \\
\hline $1 \mathrm{lfb}(8)$ & 40 & -1.47 & -0.97 & -1.68 & 0.12 \\
\hline $1 \operatorname{mbd}(6)$ & 1 & -4.37 & -1.25 & -1.44 & 0.14 \\
\hline $1 \mathrm{msi}(5)$ & 1 & -4.61 & -1.10 & -2.00 & 0.15 \\
\hline $1 \mathrm{pal}(8)$ & 4 & -2.07 & -0.25 & -1.92 & 0.14 \\
\hline $1 p d o(8)$ & 1 & -3.79 & -0.90 & -1.33 & 0.20 \\
\hline $1 \operatorname{ptq}(7)^{*}$ & 595 & 0.19 & -0.81 & -1.35 & 0.15 \\
\hline 1r69(2) & 1 & -2.90 & -1.23 & -1.72 & 0.16 \\
\hline 1ris $(5)$ & 12 & -1.75 & -0.57 & -1.65 & 0.18 \\
\hline $1 \operatorname{tul}(5)$ & 1 & -3.40 & -0.31 & -1.52 & 0.16 \\
\hline $1 \mathrm{vls}(3)$ & 1 & -2.78 & -0.62 & -1.82 & 0.19 \\
\hline 1who(6) & 1 & -3.56 & -0.21 & -0.79 & 0.19 \\
\hline $2 \operatorname{acy}(6)$ & 1 & -4.75 & -0.25 & -1.74 & 0.15 \\
\hline $2 \operatorname{gdm}(5)$ & 1 & -3.55 & -0.07 & -1.24 & 0.13 \\
\hline $5 \operatorname{pti}(3)$ & 2 & -3.25 & -0.25 & -0.81 & 0.07 \\
\hline Total/average & $17 / 23$ & -3.44 & -0.54 & -1.65 & 0.16 \\
\hline
\end{tabular}


Table G: Comparison with Chebyshev-Expanded hydrophobic potential (CHP) for the decoy sets 4state_reduced and lmds. The data separated by colons are our score : CHP score

\begin{tabular}{|c|c|c|c|c|c|}
\hline target & Rank 1 & $Z$-score & target & Rank 1 & $Z$-score \\
\hline $1 c t f$ & $1: 1$ & $-3.53:-2.9$ & $1 \mathrm{ctf}$ & $1: 1$ & $-3.42:-3.5$ \\
\hline 1r69 & $1: 2$ & $-3.68:-2.4$ & $1 \mathrm{fc} 2$ & $409: 9$ & 0.91:-2.1 \\
\hline $1 \mathrm{sn} 3$ & $1: 3$ & $-2.52:-2.1$ & ligd & $1: 1$ & $-2.87:-2.8$ \\
\hline 2 cro & $2: 60$ & $-3.01:-1.3$ & 1shf-A & $1: \mathrm{n} / \mathrm{a}$ & $-2.90: \mathrm{n} / \mathrm{a}$ \\
\hline $3 i c b$ & $1: 10$ & $-2.26:-1.6$ & $2 \mathrm{cro}$ & $1: 250$ & $-3.42:-0.4$ \\
\hline $4 p t i$ & $5: 62$ & $-2.51:-1.7$ & 2ovo & $16: 7$ & $-1.67:-2.2$ \\
\hline $4 \mathrm{rxn}$ & $4: 6$ & $-2.71:-2.2$ & $4 p t i$ & $6: 32$ & $-2.24:-1.4$ \\
\hline
\end{tabular}

Table H: Comparison with SOLV and FRST. The data separated by colons are our score : SOLV score : FRST score

\begin{tabular}{l|lllll}
\hline decoy set & Rank 1 & $Z$-score & $\log P B 1$ & $\log$ PB10 & F.E. \\
\hline 4state_reduced & $4 / 7: 1 / 7: 7 / 7$ & $-2.89:-1.7:-4.4$ & $-1.19:-0.51:-1.62$ & $-1.88:-1.78:-2.31$ & $0.28: 0.28: 0.43$ \\
lattice_ssfit & $6 / 8: 4 / 8: 8 / 8$ & $-4.06:-3.2:-6.7$ & $-0.41:-0.37:-0.58$ & $-1.55:-1.20:-1.29$ & $0.12: 0.10: 0.10$ \\
lmds & $4 / 8: 2 / 8: 6 / 8$ & $-2.75:-1.2:-3.5$ & $-0.48:-0.49:-0.48$ & $-1.36:-1.46:-1.44$ & $0.14: 0.15: 0.14$ \\
\hline
\end{tabular}




\section{Figure Caption}

Figure 1. A schematic diagram to show the quantities involved in the calculation of of $M_{i j k}$

Figure 2. A schematic diagram to show the calculation of $M_{i j k}$ for equal sized spheres. Fig.2a corresponds to the limiting case $M_{i j k}=0.5$ whereas Fig.2c presents $M_{i j k}=1$ upper limit. The Fig.2b is intermediate between the above mentioned limits, namely $M_{i j k}=0.64$

Figure 3. A plot to compare effective number of neighbours to a residue with its actual buried area obtained by using GETAREA 
This figure "figure1.png" is available in "png" format from: http://arxiv.org/ps/q-bio/0606031v1 
This figure "figure2.png" is available in "png" format from: http://arxiv.org/ps/q-bio/0606031v1 
This figure "figure3.png" is available in "png" format from: http://arxiv.org/ps/q-bio/0606031v1 